\section{La biblioteca}

\section{Una vía para mejorar la calidad del aprendizaje en ciencias sociales.}

Aproximación Multidisciplinar a las problemáticas sociales: Una vía para mejorar la calidad del aprendizaje en ciencias sociales.

Sandra Patricia Rodríguez Investigadora, Piedad Ramírez Pardo, Asesora. Sociedad Colombiana de Pedagogía SOCOLPE. Bogotá, D.C., Septiembre 1999. Centro de Documentación IDEP: 176 Págs.

Este proyecto tomó como punto de partida los resultados de la investigación: El Fenómeno de la pobreza visto desde la representación social: Una propuesta pedagógica y didáctica, que permitió identificar los contenidos del campo de representación social de la pobreza y establecer los lineamientos para el proyecto Aproximación multidisciplinar a las problemáticas sociales una vía para mejorar la calidad del aprendizaje en ciencias sociales, propuesta pedagógica y didáctica que hace posible los diferentes niveles de profundización, comprensión e indagación de la realidad si se promueve la mirada desde las ciencias sociales. Se realizaron contactos con el colegio Distrital Panamericano, donde se expuso el proyecto a la coordinadora y luego a los profesores del área de Sociales. Este proyecto elaboró una Guía de Trabajo para el maestro de Ciencias Sociales que incluye Fundamentos pedagógicos, elementos de la propuesta didáctica, los cambios en los contenidos de campo de representación, proceso metodológico y guías de trabajo para cada tema específico.

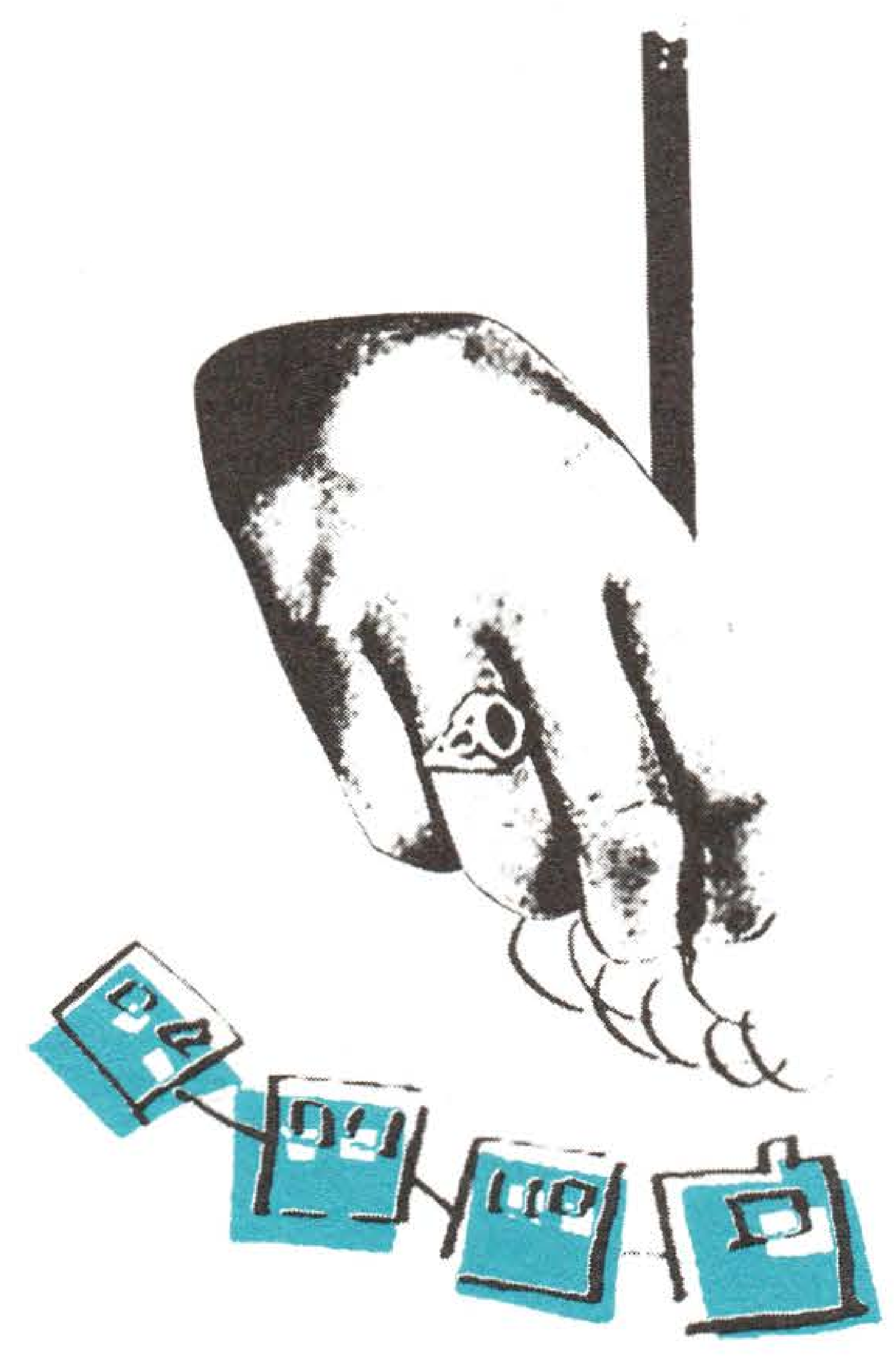

\section{Palabras clave: realidad, calidad de aprendizaje, ciencias sociales, representaciones sociales.}

El documento consta de seis capítulos entre los que se destacan: La comprensión de la realidad; Fundamentos de la propuesta pedagógica y didáctica; Exploración y reconstrucción de los significados; Dimensiones del contexto educativo y Ciencias sociales y cambio representacional. Esta articular las diversas dimensiones utilizadas para analizar las representaciones sociales que confluyen en el acto pedagógico como son, la información, el contenido del campo de representación y las actitudes, es decir las formas de comprensión de la realidad social elaboradas por los estudiantes con los aportes de la Ciencias Sociales. 


\section{Representación sobre la Evaluación de la Calidad de Gestión de las Instituciones Educativas de Bogotá}

Luis Enrique Orozco, Investigador principal, Angélica Rodríguez, Juan Carlos Alonso, Nadia López Téllez, Oswaldo Heredia, Asistentes de investigación, Universidad de Los Andes, Bogotá, Junio de 1999. Centro de Documentación IDEP.

\section{Palabras clave: Calidad, evaluación, representaciones, pedagogía popular.}

Esta investigación parte de las teorías de los autores Anthony Giddensy Jerome Brunner quienes contribuyen con un marco explicativo para la comprensión de la realidad educativa como práctica social e igualmente contribuyen a la generación de las categorías que estructuraron el análisis, es decir, las prácticas referidas al yo, las prácticas sedimentarias y las prácticas potenciales. La investigación busca enriquecer la teoría existente sobre la calidad de la educación y su evaluación a partir del análisis de las representaciones sobre calidad, evaluación y gestión de diferentes agentes educativos de Bogotá - docentes, directivos, supervisores- así como la realización de una serie de indicadores como elementos para un modelo de evaluación de las instituciones educativas.
Metodología: Se llevaron a cabo sesenta entrevistas a actores que desempeñaban distintos cargos- rectores, coordinadores y docentes- en 8 planteles educativos, 4 oficiales y 4 privados, con el fin de observar sus diferentes perspectivas. También se entrevistaron supervisores de la Secretaría de Educación del Distrito Capital para ampliar la información, socializar los hallazgos parciales y hacer una construcción y negociación colectiva de conocimientos. Se realizó una revisión documental de los PEI de los planteles como complemento al análisis de la gestión de la práctica educativa.

A partir de la información recolectada, se generaron unas categorías de análisis que dieron cuenta de los significados alrededor de prácticas educativas concretas. Así, el análisis de las prácticas referidas al yo, reveló la importancia de la autobiografía personal en la constitución de las Representaciones sobre calidad: La importancia de categorías tales como la vocación docente, el sentimiento de ser un agente interrogador, el sentimiento de ser un agente transformador, la identificación tanto positiva como negativa con experiencias personales en la vida laboral y estudiantil se hizo evidente. 
Las representaciones sobre las prácticas potenciales que comprendian la dimensión ideal de la vida escolar, expresaron que la calidad se asocia: a) con un perfil docente específico tanto a nivel personal como profesional, b) con los objetivos de formación determinados y llevados a cabo por las instituciones educativas y c) con la necesidad de un equilibrio entre la formación académica y la dimensión valorativa y afectiva a nivel institucional y personal. El estudio incluye capítulos entre los que se destacan: Hacia una Comprensión de las Representaciones de los Agentes sobre Calidad, Evaluación y Gestión de las Instituciones Educativas.; Elementos para un Modelo de Evaluación de la Calidad y de La Gestión en las Instituciones Educativas.

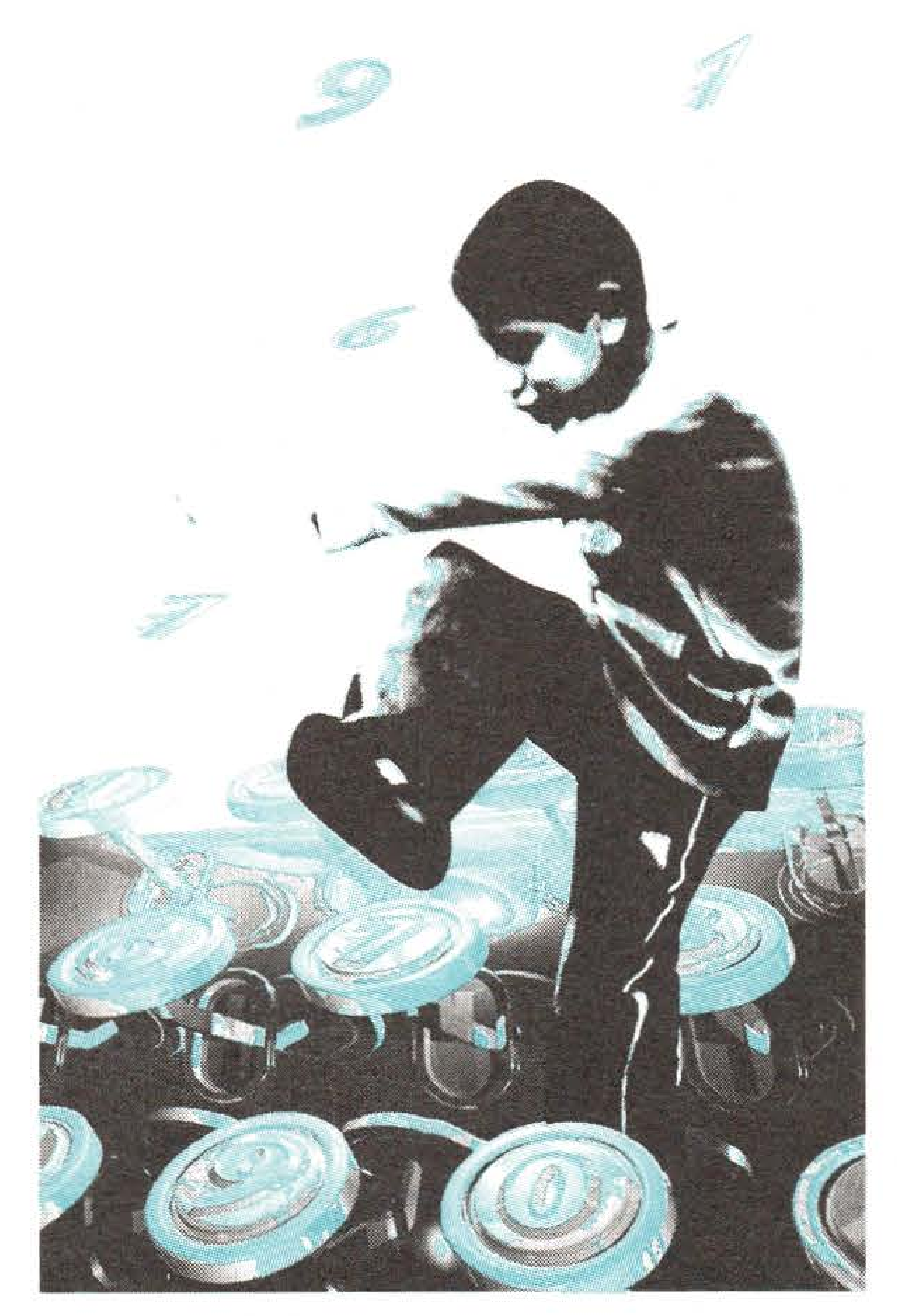




\section{Equidad de género y calidad de la educación}

Ana Rico de Alonso, Angélica Rodríguez, Juan Carlos Alonso, Nadia López, Sonia Castillo, Carlota Alméciga, Pontificia Universidad Javeriana, Facultad de Ciencias Políticas y Relaciones Internacionales. Bogotá, D.C. noviembre 18 de 1999, Centro de Documentación IDEP: 196 págs.

Este proyecto examina los diferentes procesos que determinan la calidad de la educación, haciendo énfasis en el acercamiento a los micro escenarios escolares donde se desarrollan relaciones, creencias y comportamientos que marcan la apropiación en la escuela de valores, normas y pautas relativas al género. Para ello tres planteles educativos abrieron sus puertas para permitir la construcción de un conocimiento que alimentara el debate en torno a ello: Colegio Menorah (Femenino), Colegio Monseñor Bernando Sánchez (Mixto) y el Instituto Técnico Central (Masculino. En cuanto al contenido del documento se destacan los siguientes temas: Fines y funciones sociales de la educación; Educación y currículo oculto; Género y equidad en la educación; Calidad de la educación;

El estudio busca establecer las condiciones de equidad de género en la calidad de los procesos educativos que viven alumnos y alumnas de tres planteles de la ciudad capital (Masculino, Femenino, Mixto), tomando en consideración a los estudiantes de ambos géneros, el plantel, y las relaciones que se construyen dentro del aula en las áreas de matemáticas, lenguaje y sociales. Se pretende así, profundizar en la comprensión de estos fenómenos, para el establecimiento de recomendaciones de acción que orienten las políticas de formación de docentes del Instituto para la Investigación Educativa y el desarrollo pedagógico - IDEP.

\section{Palabras clave: género, equidad en la educación; calidad de la educación.}

Se identificaron diferencias fundamentales en los procesos, en términos de la función educadora (transmisión del conocimiento), de la pedagogía, del currículo explícito. En cuanto a la función Educadora, se concluye que la transmisión de contenidos concretos, acordes con los requerimientos de la asignatura y el grado se dan de manera diferente. En el colegio masculino una porción sustancial de la clase es liderada por el docente, quien circula a los alumnos, el conocimiento que tiêne de la materia. En el colegio femenino y en el colegio mixto coexisten diferentes modalidades, muy marcadas por el estilo personal de cada docente; algunos docente dan sus clases con evidente contenido del campo de conocimiento, en tanto otros inician un debate y lo moderan, sin que se expliciten siempre los objetivos del mismo, y sin llegar a una clara síntesis que articule las posturas en el debate con la especificidad de la asignatura. Otros docentes delegan en el estudiante el desarrollo de la sesión, bien sea distribuyendo textos, o asignando ejercicios, con una circulación del/a docente en una relación más de supervisión que de transmisión. 
Respecto a las estrategias pedagógicas el estudio concluye con la siguiente caracterización: cátedra magistral, debate, trabajo en grupo, y asignación de tareas para desarrollar fuera del aula, dentro de combinaciones diferentes. En relación con el currículum explícito, es evidente que existe una correlación entre la intensidad de la transmisión de conocimiento y la presencia de un currículum explícito, en términos de contenidos que deben cubrirse en cada grado. Si bien todos los planteles tienen unos criterios en términos de logros, que son base para la evaluación, no se capta tan directamente el programa del curso. Entre los factores asociados con una alta calidad académica, expresada como una función conjunta de procesos y resultados se encuentran los siguientes: Factores endógenos del plantel; Factores exógenos; Proyecto de vida de los estudiantes y Percepciones y creencias de los/as directivos y docentes.

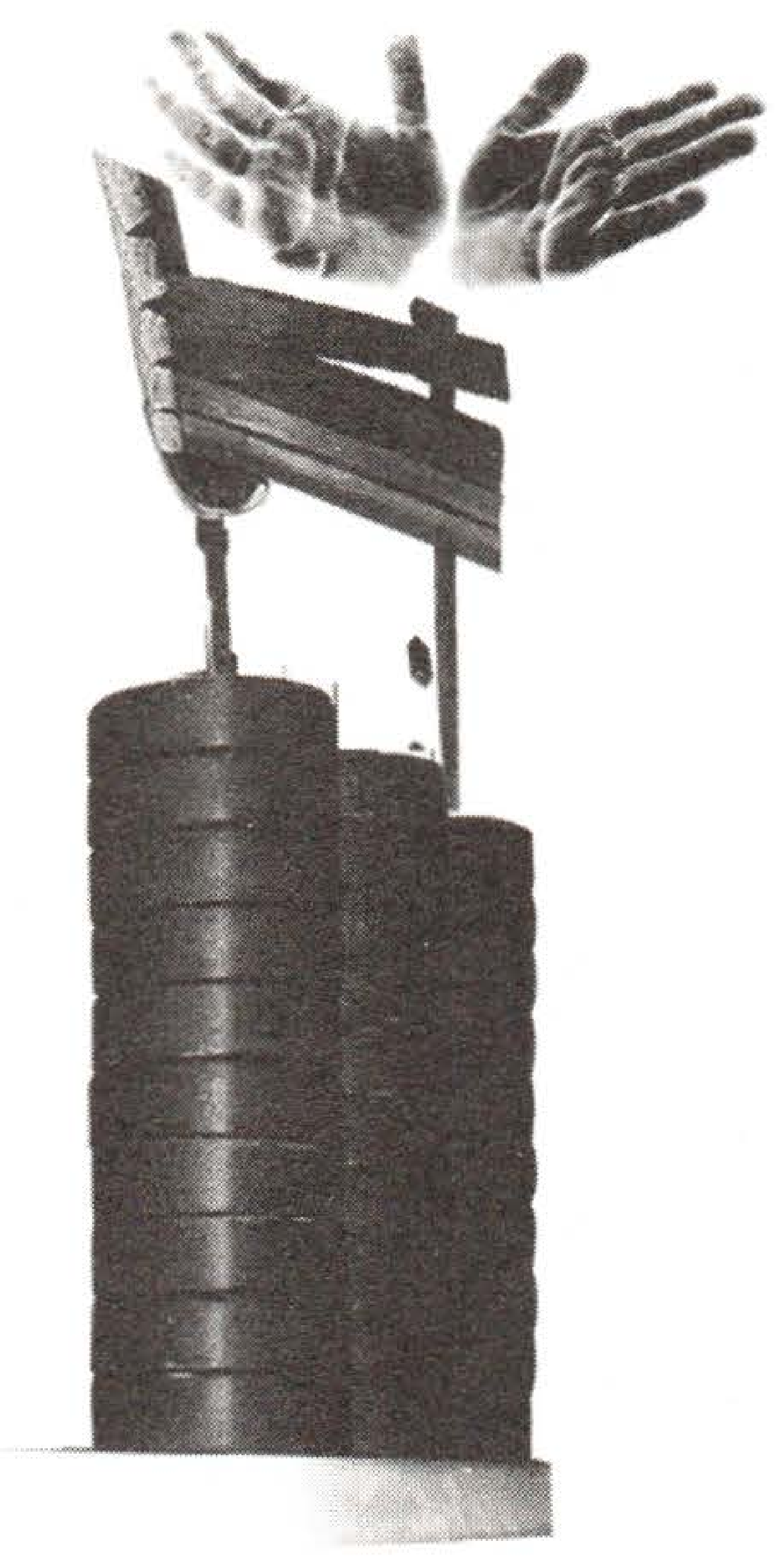




\section{Los jóvenes, su participación, sus aprendizajes y la calidad de la educación}

Elsa Rodríguez, Investigadora; María Leonor Charria y Nohemí Abadía, Coinvestigadoras, Fundación Universitaria Monserrate, Bogotá, D.C. Noviembre de 1999, Centro de Documentación IDEP: 90 págs.

Esta investigación está basada en el siguiente interrogante: ¿En qué medida los procesos participativos que se constituyen en mediadores de los aprendizajes que realizan los jóvenes en sus grupos de pares, se relacionan con el tipo de participación que incentiva la escuela en los aprendizajes formales? La pesquisa se realizó en dos instituciones oficiales (Colegio de los Periodistas y Unidad Básica Rafael Uribe Uribe), con jóvenes entre 12 y 16 años, de estrato 2 y 3, más sus respectivos maestros.,

El informe incluye apartes entre los que se destacan: La explicación de lo que significa ser joven y cómo los jóvenes experimentan los aprendizajes en grupos de pares; El análisis de los jóvenes y sus grupos dentro de los aprendizajes escolares; la identificación de las características de los jóvenes dentro de un ambiente escolar. Concentrándose en el examen de las vivencias de los jóvenes, explica en especial aquellas que suceden en los grupos de pares tanto en el ambiente escolar como extraescolar y que tienen que ver con las formas participativas que emplean en sus procesos de socialización. Además analiza cómo estas vivencias inciden en las maneras en que los jóvenes abordan sus aprendizajes formales en el proceso escolar, los cuales pueden ser reconocidos, ignorados o subvalorados por los docentes. La investigación parte de un enfoque cualitativo y corresponde al modelo hermenéutico, siguiendo una perspectiva crítica-social. Teniendo en cuanta esta perspectiva, el estudio se inició con una revisión bibliográfica y se construyó el archivo descriptivo teórico que se utilizaría en el transcurso de toda la investigación. Posteriormente se seleccionaron las fuentes primarias y se utilizaron instrumentos de investigación (elaboración de cartas, diarios de campo, dinámicas grupales, video foros, entrevistas individuales, cuestionarios, entrevistas grupales y conversatorios con alumnos y docentes), los cuales privilegiaron la observación no participante. Con la información aquí adquirida, los investigadores elaboran un archivo descriptivo interpretativo de la información primaria.

Palabras clave: jóvenes, grupos de pares, participación, aprendizaje, escuela, calidad educativa 


\section{Equidad educativa y gobierno territoriales en Colombia: ¿Inclusión o segmentación?}

Catalina Turbay y Ángela Bacca (2008) Equidad educativa y gobierno territoriales en Colombia: ¿Inclusión o segmentación? Aique Educación, Buenos Aires.

Catalina Turbay Restrepo

Durante las décadas de los años 1980 y 1999 en sus inicios, las políticas sociales en la región latinoamericana estuvieron signadas por reformas que, entre otras, promovieron la descentralización de los servicios educativos, en algunos casos como alternativa para reducir la ineficiencia estatal y, en otros, o al tiempo, como alternativa para responder mejor a los requerimientos de los territorios y comunidades. Es en este marco, que se realizó un estudio exploratorio regional en cuatro países de América Latina (Argentina, Colombia, Chile y Perú), buscando establecer cuál es el papel de los niveles intermedios de gobierno, es decir, de los gobiernos que median entre la nación y las instituciones educativas, en la construcción de equidad educativa.

Colombia no fue ajena a la dinámica reformista de los gobiernos, iniciando a partir de los ochenta un proceso descentralizador que vendría a ser reforzado por la Constitución de 1991, hito reformista con el que se sembraron las semillas de tensión entre las tendencias proclives al estado social de derecho y la descentralización que ella consagraba, y los partidarios de una contrarreforma que, en no pocos casos, se impulsó posteriormente. En este marco institucional y político, se formularon diversos dispositivos normativos

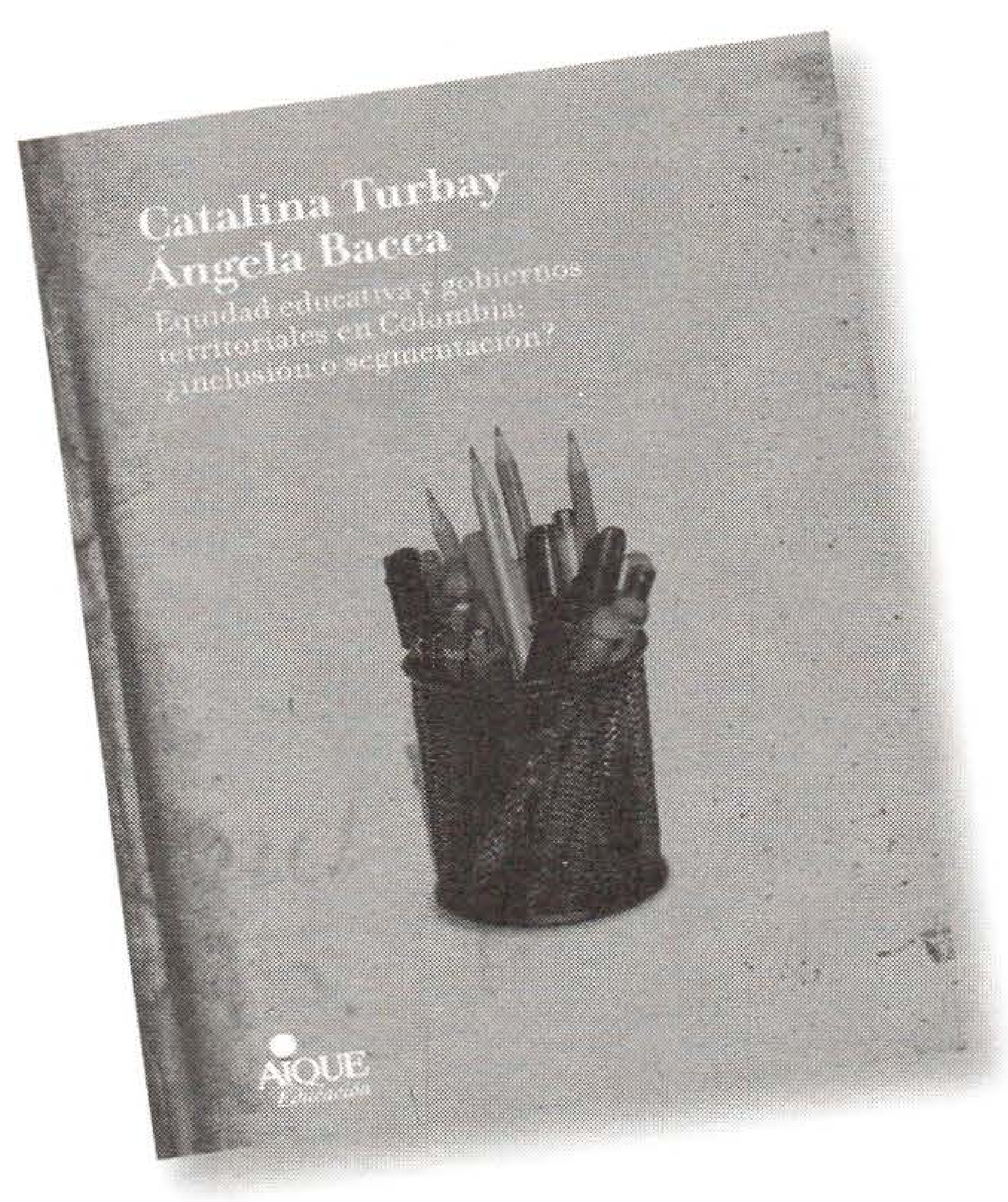

que regularan la educación - iconcebida como derecho o como servicio? - bajo nuevos parámetros, los cuales, en principio, deberían ser menos centralistas y promover el derecho, pero que, en efecto, han estado signados por la tensión mencionada.

Hasta ahora, las reformas hacia la descentralización de los servicios sociales, y, en particular, de la educación, desarrolladas en Colombia durante las últimas décadas han sido objeto de análisis y evaluaciones, en especial la Ley 60 de 1993 y la Ley 715 de 2001, sin que se haya examinado con detalle cómo se están configurando las cadenas de mediación e intermediación de los diferentes niveles de gobierno, ni el papel efectivo que tienen, en nuestro caso, las Secretarias de Educación de las entidades territoriales certificadas, para el logro de la equidad en el sector. Es decir, que no obstante los argumentos a favor del papel decisivo de los gobiernos cercanos a las realidades locales y a las comunidades en la construcción de sistemas educativos más justos, en Colombia no se había adelantado estudio alguno que se aproximara en profundidad al papel efectivo de los gobiernos subnacionales, sus alcances y limitaciones como agentes de la política para la equidad educativa. 
trabajo busca llenar este vacío, en procura de comprender y caracterizar las reformas recientes del sector, desde la comprensión de las cadenas de intermediación que se han configurado en el marco de las mismas. Para indagar sobre el papel que pueden tener los organismos del nivel intermedio en la búsqueda de la equidad, atendiendo a este contexto que se expresa de manera singular en los territorios, y conforme al marco teórico general elaborado para todos los países, el estudio centró su atención, tanto en el plano formal como factual, en el quién y en el qué de los gobiernos intermedios. El quién en lo que respecta a las modalidades de organización y la articulación de estos actores dentro del sistema; el qué por su parte en lo que atañe a las competencias asignadas y las funciones que efectivamente asumen las organizaciones de los niveles intermedios.

¿Pueden los gobiernos sectoriales del orden nacional, intermedio y local responder a estos desafíos? En un contexto de reformas administrativas, ¿quiénes son estos organismos? ¿Cuáles sus competencias y qué hacen? ¿Qué papel desempeña la construcción de equidad educativa en las agendas gubernamentales? El estudio exploratorio del caso colombiano que se presenta quiso avanzar en esta vía y aproximarse en forma cualitativa a una mirada en profundidad de lo que sucede en estas instancias, para de ahí derivar elementos que orienten estudios posteriores.
El libro recoge los resultados de la investigación adelantada entre 2005 y 2006 en el marco de un proyecto comparado sobre institucionalidad y gobiernos educativos intermedios apoyado por la Fundación Ford. Metodológicamente se abordó mediante el estudio de casos de dos organizaciones gubernamentales del sector educativo: la capital y un municipio o provincia (Bogotá y Duitama, para el caso colombiano). El estudio de los casos pone en evidencia los contrastes, las capacidades y recursos desiguales que tienen los gobernantes de los entes territoriales cercanos a las realidades locales, a las escuelas y a las comunidades, en la construcción de sistemas educativos más justos, producto de la heterogeneidad del país y su segmentación histórica.

La estructura expositiva del libro abarca cuatro capítulos. En el primero se presentan los principales referentes conceptuales sobre equidad y formas de gobierno que orientaron el trabajo. El segundo capítulo, recoge los principales hallazgos derivados del análisis de los marcos institucionales vigentes, partiendo de una información básica sobre la estructura de gobierno en Colombia haciendo una ubicación histórica de los marcos normativos generales, analizando sus alcances descentralizadores en la asignación efectiva de competencias a los gobiernos de los diferentes niveles del sistema y el lugar que ocupa la equidad, se presenta también el resultado del análisis de la normativa nacional para establecer cuáles son las competencias que en términos formales se otorgan a los diferentes niveles de gobierno, a la luz de las categorías construidas al 
efecto para los aspectos pedagógicos-curriculares y administrativos-financieros y se formula una reflexión sobre las reformas y los alcances y limitaciones metodológicas de recurrir al examen de las normas como fuente para dar cuenta de intervenciones por la equidad educativa.

Del análisis de los casos de Bogotá y Duitama y del caso del CADEL de ciudad Bolívar que se incluyó para efectos del estudio de la capital dada su organización administrativa en localidades para atender a las instituciones educativas, se ocupa el tercer capítulo. Comienza con una presentación de aspectos metodológicos y contextualiza los casos desde variables sociodemográficas y educativas, analizando los indicadores sectoriales desde la equidad educativa y haciendo una comparación del desarrollo de las dos ciudades, contrastada con los promedios nacionales. Posteriormente se presentan los hallazgos referidos a las características constitutivas (el quién y cómo) de los gobiernos educativos de Bogotá y Duitama (estructuras orgánicas; capacidades existentes; modelos de autoridad, autonomía y participación en el trazado de políticas, y formas de actuación política), tras lo cual se exponen los hallazgos relativos a las funciones que desempeñan las secretarías de Educación en los ámbitos pedagógicocurricular y administrativo-financiero. El capítulo cierra con un análisis de la equidad educativa en el quehacer de las organizaciones de los niveles intermedios estudiadas $\mathrm{y}$ las posibilidades efectivas que tienen para incidir a favor de la garantía del derecho a la educación.
El libro culmina con un capítulo donde se exponen las principales conclusiones derivadas del estudio y se señalan algunos de los principales desafíos que debe asumir el gobierno nacional y el país si, efectivamente, se desea avanzar en la construcción de un sistema educativo más equitativo y reducir las brechas territoriales existentes, fortaleciendo las capacidades de las regiones así como focalizando las políticas en temas fundamentales que aparecen rezagados al examinar la equidad educativa, como la calidad y la pertinencia.

Un capítulo síntesis de este estudio, se presenta también en el libro de la colección que recoge el estudio comparado regional, coordinado por Silvina Gvirtz "Equidad y niveles intermedios de gobierno en los sistemas educativos. Un estudio de casos en la Argentina, Chile, Colombia y Perú" Si bien se trata de una investigación exploratoria, no cabe duda que los hallazgos de este estudio, habrán de ser considerados para avanzar en la investigación y trazado de las políticas públicas en educación.

El tema abordado en los dos libros resulta de vital importancia en el contexto de las transformaciones del Estado y los desafíos que para los diferentes niveles del mismo, representa construir sociedades más justas y, dentro de éstas, equidad educativa, ofreciendo a la vez alternativas metodológicas y conceptuales para su análisis, junto con opciones para las políticas públicas. 


\section{"Equidad y niveles intermedios de gobierno en los sistemas educativos. Un estudio de casos en la Argentina, Chile, Colombia y Perú"}

Silvina Gvirtz (coord.) Buenos Aires, Aique, 2008 Autores: Silvina Gvirtz, Gustavo Dufour (con la colaboración de Fernanda Page y Natalia Chami), Marcela Román, Hugo Díaz, Néstor Valdivia, Rodrigo Lajo, Catalina Turbay, Ángela Bacca.

\section{Marina Larrondo ${ }^{l}$}

La producción de literatura sobre política educativa y reforma educativa en América Latina ha sido prolífica. En las últimas dos décadas -y en coincidencia con la puesta en marcha de procesos de reforma de los sistemas educativos- las universidades, organismos internacionales, fundaciones, organizaciones no gubernamentales han apoyado o llevado adelante investigaciones e informes. También es abundante la producción y la literatura sobre los distintos aspectos del problema de la equidad educativa, una prioridad en la agenda latinoamericana. Por último, desde estas instancias se han debatido -y se debaten- los conceptos y abordajes utilizados para aprehender la realidad sociopolítica de la educación en la región.

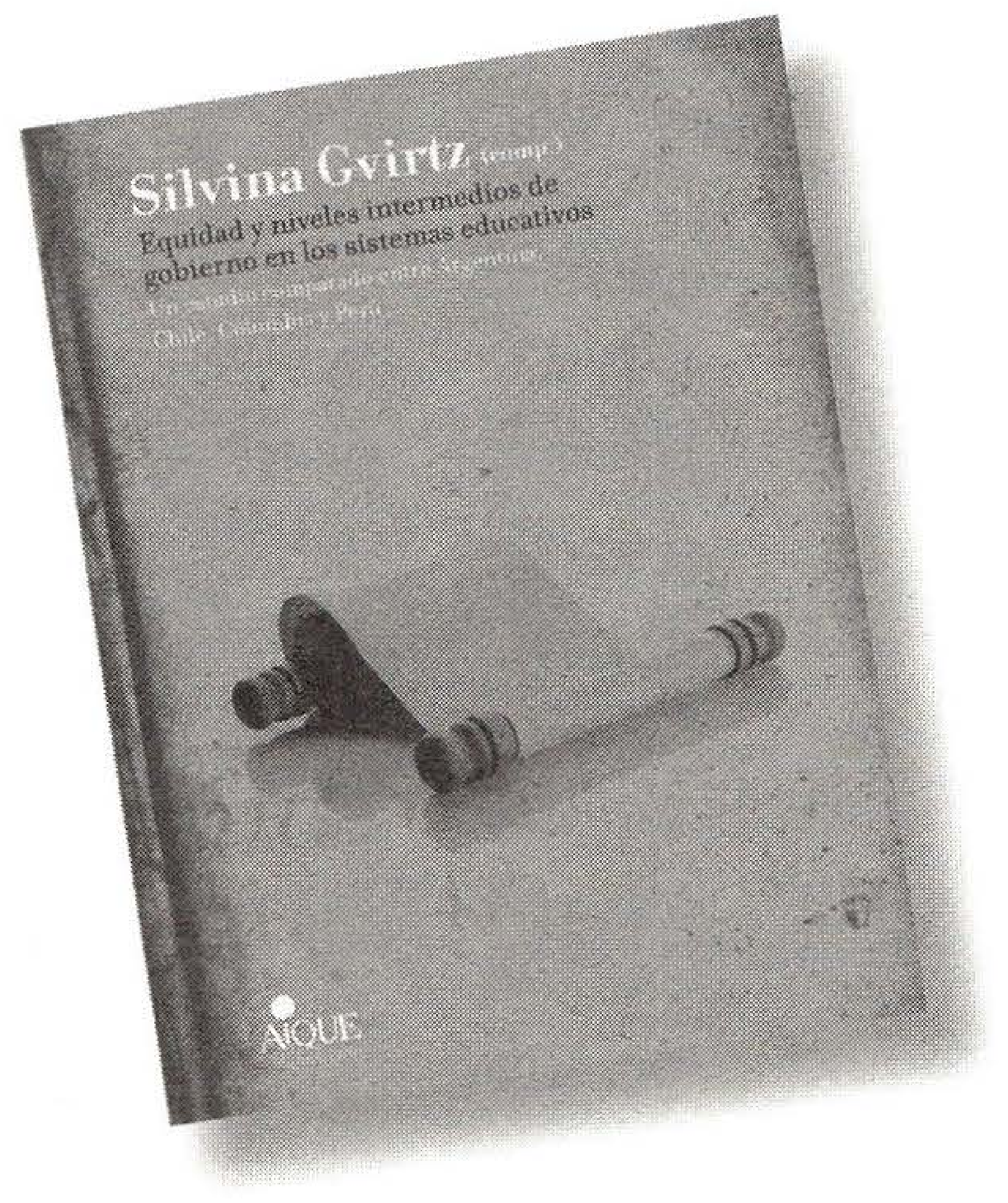

Sin embargo, no es arriesgado decir que en dicha producción hubo un gran ausente: los niveles intermedios de gobierno del sistema educativo. Es decir, aquellos actores que se ubican entre los niveles centrales de toma de deccisiones - ministerios nacionales y/o provinciales- y las escuelas. Si esta "ausencia" se debe a una subestimación de su función (ser "ejecutores de políticas"), este libro mostrará lo contrario. Este libro es ni más ni menos que la primera contribución (o una de las primeras) al estudio específico de estos actores; es el resultado de una investigación empírica, financiada y apoyada por la Fundación Ford. Su principal fortaleza es que brinda una aproximación descriptiva y rigurosa

1 Socióloga (UBA); Especialista en Educación (Universidad de San Andrés). Docente (asistente) de la Universidad de San Andrés. 
sobre los niveles intermedios de gobierno del sistema educativo, para preguntarse por las potencialidades y los límites de estos actores en el logro de la equidad. En síntesis, abre un campo problemático y lo da a conocer, permitiendo iniciar una discusión sobre el tema.

En la investigación, la pregunta por los niveles intermedios parte de un supuesto: los nivelesintermedios no son simples "correas de transmisión" en el sistema educativo. Se trata de actores que inciden tanto en la implementación de la política educativa (y por lo tanto en la política educativa misma) como en la vida cotidiana de las escuelas. Los modos y alcances de dicha incidencia es lo que se explora en cada caso nacional (Argentina, Chile, Colombia y Perú), como así también en qué medida contribuyen o no en el logro de la equidad.

Desde el punto d e vista temático, el libro está dividido en una introducción, cinco capítulos y conclusiones. El primer capítulo expone el marco teórico de la investigación. Aquí, la construcción de un marco teórico ha presentado un doble desafío para el equipo de autores. En primer lugar, porque se trata de un tema poco estudiado previamente en la región. En segundo lugar, porque dicho marco teórico debía permitir conceptualizar actores en sistemas educativos distintos, es decir, debía permitir un enfoque comparativo. Los capítulos 2, 3, 4 y 5 reflejan los resultados de la investigación en cada uno de los casos nacionales. En cada capítulo se describe en primer lugar los rasgos de cada sistema educativo explicitando qué lugar ocupan en cada uno estos niveles. En segundo lugar, se describen las estructuras de autoridad de los niveles intermedios y su modo funcionamiento: qué, quiénes y cómo se decide tanto en las cuestiones administrativofinancieras como pedagógicas. Ahora bien, como mencionábamos, el análisis no tiene sólo una impronta meramente descriptiva sino que está orientada por la pregunta sobre la equidad. De este modo, cada caso nacional va acompañado por una serie de reflexiones sobre las potencialidades y límites de estos actores para contribuir al logro de la equidad, y se brindan algunas recomendaciones para avanzar en este sentido.

El transcurso de la investigación ha permitido concluir a los autores que más allá de los obstáculos normativos, presupuestarios y de organización que enfrentan los niveles intermedios, ellos tienen la potencialidad de generar acciones a favor de una mayor equidad en los sistemas educativos latinoamericanos.

La publicación de este libro es sin duda, una valiosa contribución al conocimiento de los sistemas educativos y su arquitectura institucional; y también al estudio del problema de la equidad. Tiene el mérito de ser una investigación pionera que abre las puertas para futuras investigaciones -muy necesarias- sobre el tema; y se destaca especialmente el esfuerzo de construir elementos conceptuales que permitan la comparación entre países. Seguramente, será más que bienvenido por los tomadores de decisiones en política educativa, por investigadores y estudiantes y por los propios sujetos de la investigación. 


\section{Hábitat escolar, más allá de la infraestructura educativa}

\section{Arq. Carlos Benavides Suescún}

Secretaría de Educación, Alcaldía Mayor de Bogotá, Bogotá D.C. 2008 Impresión

Es admirable como están cambiando las tapias altas y el alambre de púas que caracterizaba a algunos colegios bogotanos por un ambiente arquitectónico más amable, con espacios que permiten la generación de vínculos comunitarios, que plantean nuevas formas de educar y establecen nexos de relación con la comunidad. En años anteriores muchas de las edificaciones escolares se levantaban de cualquier manera, en zonas no aptas, cercanas a las rondas de los ríos, en suelos sujetos a deslizamientos y en terrenos inadecuados y sin normas técnicas de acuerdo a su uso. Desde hace una década este panorama empezó a cambiar y, hoy por hoy, se vislumbra más alentador gracias a los esfuerzos de varias administraciones distritales ${ }^{l}$

A comienzos del siglo pasado, los colegios oficiales eran ejemplos destacados tanto por su sobresaliente nivel académico como por la calidad de su arquitectura, convirtiéndose en símbolos representativos de la ciudad; sin embargo el acelerado y desordenado crecimiento de la urbe generó un paulatino deterioro en la calidad de la escuela pública, relegando en muchos casos sus instalaciones a unas deplorables e indignas construcciones de espaldas a la ciudad, más cercanas a un centro de reclusión (incluso algunas se denominaban concentraciones escolares) que al recinto de formación que para toda la vida marcará huella en niños y jóvenes. Esta situación se ha venido modificando positiva y gradualmente en'la capital del país, convirtiéndose en una experiencia a seguir que hoy es ejemplo a nivel nacional e incluso a nivel latinoamericano.

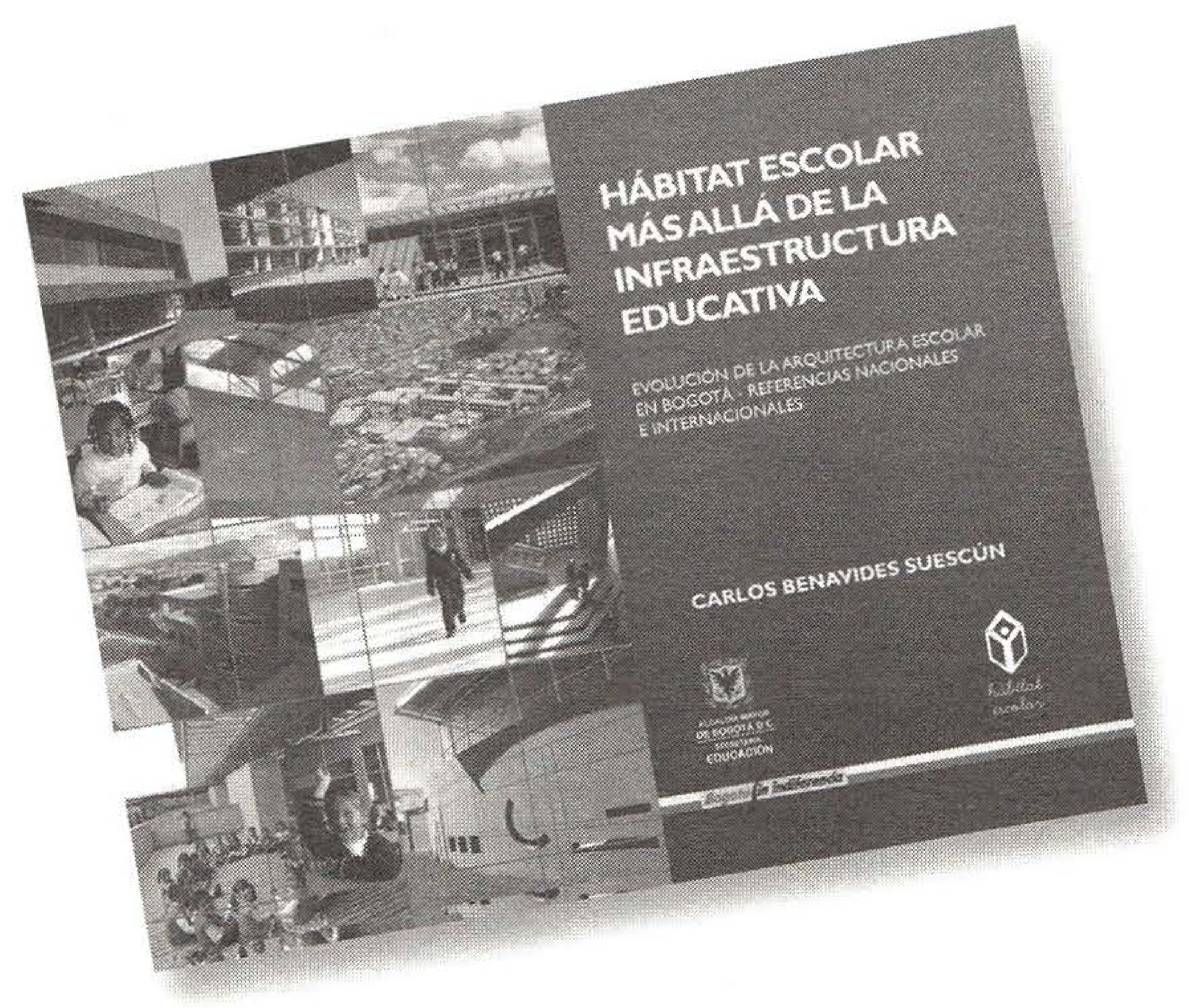

Este trascendental cambio que desafortunadamente ha tenidounalimitadadivulgaciónyungrandesconocimiento en la opinión pública por la localización mayoritaria de estos equipamientos educativos en zonas periféricas marginales, ha logrado la recuperación y el desarrollo urbanístico de sus áreas de influencia, a través de la construcción y renovación integral del parque escolar oficial, generando no solo un considerable mejoramiento en la calidad de la educación sino también en la calidad de vida de estos sectores, transformando estas tristes instalaciones, hacinadas, inseguras y aisladas de su entorno, en lugares dignos, símbolos institucionales, lugares de encuentro comunitario, generadores de espacio público y referentes obligados de arquitectura escolar de calidad. 
En la anterior administración se lograron superar con creces las metas inicialmente fijadas, para la construcción y dotación de nuevos colegios, el reforzamiento, ampliación y mejoramiento integral de una gran parte de las plantas físicas existentes, completando hoy más de dos millones de metros cuadrados construidos en las instituciones oficiales que albergan a más de un millón de estudiantes y cerca de treinta mil docentes, con instalaciones que cuentan con una gran calidad en su arquitectura, mobiliario y equipos, gracias a este esfuerzo y a la mayor asignación de recursos de inversión en la historia de la ciudad, del país y quizá de América Latina en cuatro años.

La Secretaría de Educación de Bogotá ha liderado a nivel nacional la recuperación masiva de sus plantas físicas actualizándolas a normas de seguridad y sismo resistencia, contando hoy con nuevas construcciones que permiten una total accesibilidad y uso de las instalaciones para los discapacitados, aplicando altos estándares tanto urbanísticos como arquitectónicos.

Estos logros han sido el resultado de un largo y perseverante proceso, de la continuidad de voluntades y políticas comunes, construyendo sobre lo construido, buscando un mejoramiento continuo y consiguiendo los recursos justos requeridos. Fruto del esfuerzo de la Alcaldía Mayor de Bogotá, de la Secretaria de Educación del Distrito Capital (SED) y del trabajo interdisciplinario de pedagogos, docentes, profesionales de la arquitectura y la ingeniería, técnicos y demás funcionarios, con el aporte de la comunidad educativa y el apoyo de diversas entidades e instituciones. La actual administración ha continuado este proceso de mejoramiento integral de los ambientes escolares en la capital, esperando que esta valiosa experiencia aporte como ya lo ha venido haciendo en otras ciudades, al mejoramiento y construcción de los ambientes escolares en nuestro país, que decididamente influirá en el mejoramiento de la educación, la sociedad, el medio ambiente, lo urbanístico y lo arquitectónico, preservando nuestro patrimonio histórico y cultural.

Las realizaciones alcanzadas generan una gran responsabilidad no solo para poder completar y continuar este proceso irreversible, construyendo ese mejor hábitat escolar para las generaciones venideras, sino también heredando y asumiendo la preservación y conservación de esta nueva y bella infraestructura que si bien es primordialmente para el uso de la comunidad educativa, nos pertenece a todos.

El libro Hábitat escolar, más allá de la infraestructura educativa recoge las experiencias de lo que ha sido este proceso de transformación enfatizando en la necesidad de superar el alcance del concepto físico de la infraestructura educativa adoptando uno mucho más amplio como es el de hábitat escolar. La investigación comprende seis capítulos, que contextualizan y abordan la evolución del espacio escolar como respuesta a las transformaciones en los modelos pedagógicos y a las cambiantes ayudas educativas y tecnológicas, presentando y analizando las distintas herramientas de apoyo para la planificación, diseño, construcción y mantenimiento de la infraestructura escolar.

Al final se plantean algunas perspectivas de lo que podrá ser el futuro del hábitat escolar. 
En primera instancia se resume lo que ha sido el desarrollo histórico de los ambientes educativos desde los bellos e impactantes foros, ágoras y pórticos, de la Grecia y Roma antiguas, sitios de encuentro e intercambio de saberes, luego los claustros religiosos, las aulas magistrales del estricto sistema Lancasteriano en la época de la revolución industrial, la influencia de los cambios pedagógicos en los ambientes educativos con la Escuela Nueva y las propuestas de Decroly, Piaget, Montessori, presentando referentes trascendentales de la arquitectura escolar moderna como las propuestas innovadoras de Hans Sharoun, Richard Neutra, hasta los proyectos de avanzada del holandés Herman Hertzberger quien con su arquitectura, ha sido uno de los que mejor ha sabido interpretar y aportar a los procesos se enseñanza y aprendizaje.

Con respecto a nuestro país, se incorporan y sintetizan apartes del libro de Rafael Maldonado (q.e.p.d.) Historia de la arquitectura escolar en Colombia, desde la colonia y el primer centro educativo, el Colegio seminario de San Bartolomé, las escuelas patrióticas promovidas por Bolívar y Santander, incluyendo algunas de las edificaciones que hoy son Patrimonio Nacional como el colegio de la Merced Capuchina de Lascano Berti y el Liceo Agustín Nieto Caballero; se presenta un símbolo de arquitectura escolar como lo es el Gimnasio Moderno del norteamericano Roberto Harrington, los importantes aportes de la "Época de oro" del Ministerio de Obras Públicas en el gobierno de Alfonso López Pumarejo, con proyectos de destacados arquitectos como Leopoldo Rother, Julio Bonilla, Bruno Violi entre otros. Se incluye además el primer puesto de Fernando Martínez en la Primera Bienal de Arquitectura en Colombia en 1960, con la propuesta para el Colegio
Emilio Cifuentes en Bogotá que marco una gran influencia en la arquitectura nacional y posteriormente la creación y los trascendentales aportes del Instituto Colombiano de Construcciones Escolares ICCE.

El libro ahonda en la evolución de la arquitectura escolar oficial en Bogotá, resaltando la búsqueda inicial de proyectos de calidad arquitectónica como la propuesta republicana ganadora de uno de los primeros concursos para modelos tipológicos(1916) como el de la escuela República Argentina (hoy en funcionamiento) edificio patrimonial, presentando los distintos modelos adoptados por las administraciones Distritales, tipologías de los años 30, el modelo implementado en el gobierno de "Rojas Pinilla", la propuesta que más se ha repetido en la ciudad, la tipo "Alianza" (en la época del programa "la Alianza para el Progreso"), diseño del arquitecto y alcalde de Bogotá Jorge Gaitán Cortés; el modelo Tipo Experimental del pedagogo y arquitecto Luis Rivera Farfán a finales de los años 1960, la Tipología "Barco" en los años 1970, las propuestas del PIDUZOB (Plan Integrado de Desarrollo Urbano Zona Oriental de Bogotá) etc.; posteriormente se muestra como se fueron deteriorando las escuelas públicas al tiempo que crecía desordenadamente la ciudad y como surgen propuestas espontáneas sin los mínimos requisitos tanto pedagógicos como técnicos y como se inicia en el año 1998 una nueva etapa con una arquitectura escolar de gran calidad, que retoma los aportes de algunos de los mejores arquitectos de nuestro país y que hoy presenta esta nueva generación de colegios ejemplo y referente de equipamientos educativos, rescatando la importancia de la escuela pública como símbolo de integración urbana y constructora de ciudad. 
El segundo capítulo incorpora las relaciones entre pedagogía y arquitectura pretendiendo establecer ese puente que enriquezca las respuestas arquitectónicas originadas por las necesidades pedagógicas, a la vez que se demuestra objetivamente la relación directa entre la calidad de los ambientes escolares y la calidad de la educación. El libro incluye y analiza también normativas, estándares, recomendaciones y orientaciones para el planeamiento, diseño, construcción, mantenimiento y administración de los ambientes escolares, aportando además indicadores y referencias internacionales. La investigación no se limita al tema arquitectónico y el análisis detallado de cada uno de los distintos ambientes escolares por cuanto incorpora aspectos relacionados con los requerimientos de dotación, mobiliario, y señalización.

El estudio presenta un resumen del Plan Maestro de Equipamientos Educativos (hoy Decreto 4449 de 2006), que con su planteamiento de un sistema territorial de nodos educativos integrado y sinérgico con los demás equipamientos de la ciudad (parques, bibliotecas públicas, escenarios culturales y deportivos, ha sido referente y ejemplo para la construcción de otros planes maestros en Bogotá y que adicionalmente fija ya no recomendaciones sino estándares de seguridad, urbanísticos y arquitectónicos de obligatorio cumplimiento para la aprobación de nuevos colegios y de manera gradual hasta el año 2019 para la adecuación de los centros educativos existentes tanto oficiales como no oficiales.
También aporta a una propuesta de arquitectura escolar sostenible a través de una Guía Ambiental del Hábitat Escolar, continuando y ampliando los avances que al respecto se han incorporado en los últimos diseños de los colegios Distritales.

En el capitulo cuatro se significa la problemática común de la infraestructura escolar en Latinoamérica y el Caribe, resumiendo algunas de las recomendaciones y conclusiones de Seminarios internacionales organizados en colaboración con la UNESCO, incluyendo el III Seminario realizado en Veracruz México, en el año 2001, donde la ponencia presentada por Colombia, titulada La nueva arquitectura educacional pública en Bogotá, hacia un mejor hábitat escolar fue destacada y tomada como referente a emular en la región; también se presentan las conclusiones del encuentro organizado por Unión Internacional de arquitectos (UIA) sobre Espacios Educativos y Culturales realizado en Santiago de Chile en el año 2003.

Se incluyen también destacados ejemplos latinoamericanos como los Centros Educacionales Unificados (CEUs) de Sao Pablo, Brasil y una muestra de algunos de los recientes y más destacados proyectos de recuperación y construcción de planteles escolares en Medellín. 
Finalmente el libro presenta una muestra significativa de lo que ha sido la última generación de colegios en Bogotá, incluyendo el concurso público de amplia participación organizado a través de la Sociedad Colombiana de Arquitectos en el año 2004 para escoger varias propuestas que hoy son realidad para la elaboración del modelo de sistema de diseño para las instituciones educativas distritales.

Esta amplia investigación iniciativa y fruto de la experiencias del Arquitecto Carlos Benavides Suescún especialista en infraestructura escolar, quien ha estado vinculado a la SED desde hace más de 27 años, ha sido publicada con el patrocinio de la Alcaldía Mayor de Bogotá y su Secretaría de Educación; en la actualidad el Ministerio de Educación Nacional esta interesado en difundir a través de las Secretarías de Educación y las facultades de arquitectura de todo el país esta publicación buscando que sea un valioso aporte y referencia para la construcción y mejoramiento del hábitat escolar en Colombia. El libro fue premiado con mención en la pasada XXI BIENAL DE ARQUITECTURA EN COLOMBIA (2008), dentro de la categoría Teoría, Historia y Critica.

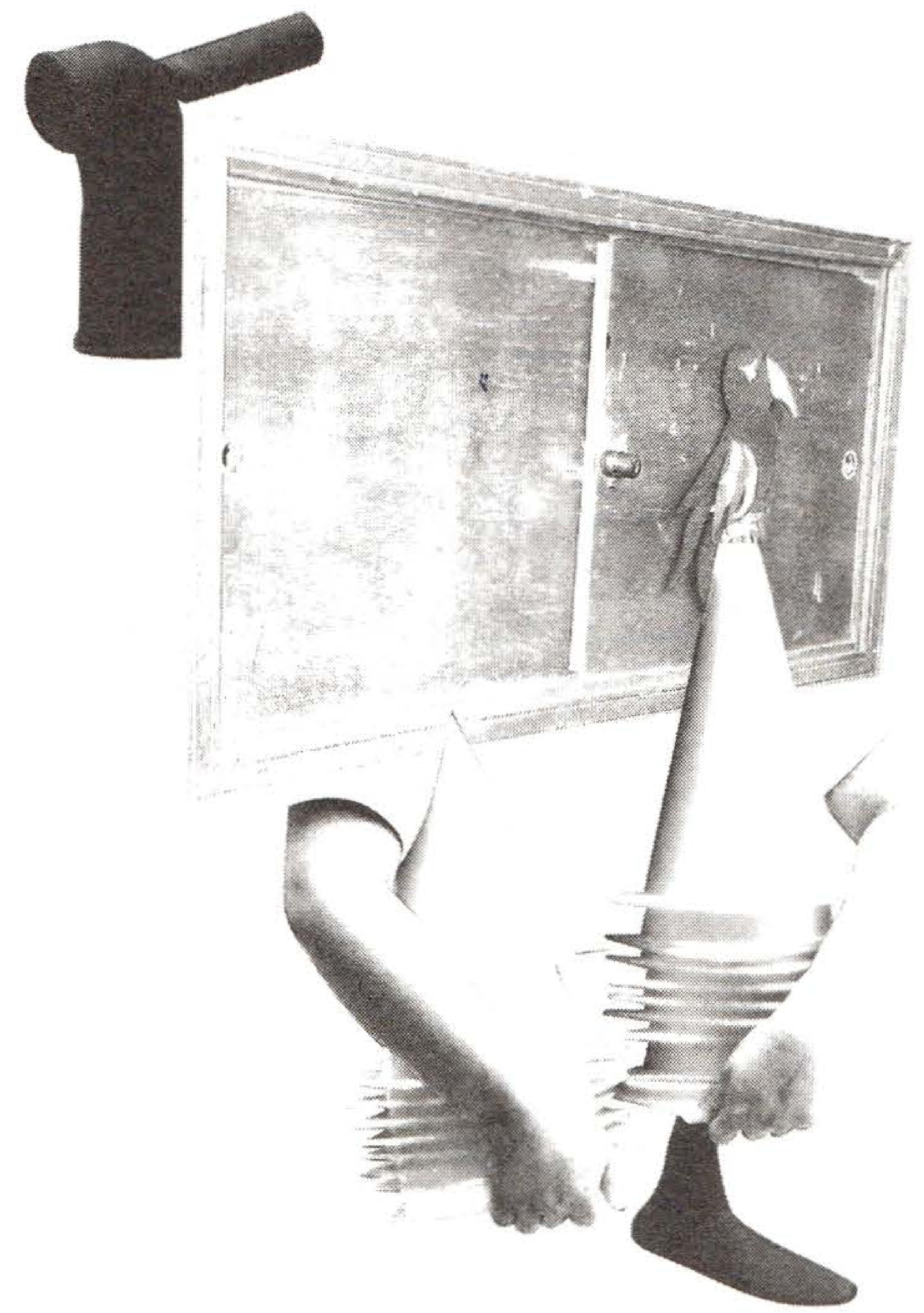




\section{Seis Ciudades, cuatro países, un derecho: análisis comparativo de políticas educativas}

Luis Eduardo Pérez Murcia, Rodrigo Uprimmy Yepes, Camilo Ernesto Castillo Sánchez, Clara Márquez Vásquez y Nathalia Carolina Sandoval Rojas. Seis Ciudades, cuatro países, un derecho: análisis comparativo e políticas educativas. ${ }^{1}$ Bogotá, D.C.: Centro de Estudios de Derecho, Justicia y Sociedad DeJusticia, Instituto para la Investigación Educativa y el Desarrollo Pedagógico IDEP, 2. Ediciones Antropos, 2007.

"Los derechos sociales, como el derecho a la educación, han provocado una división profunda entre al menos dos visiones distintas sobre la democracia y sobre los derechos humanos. De un lado, encontramos las visiones liberales individualistas radicales de autores como Von Hayek ${ }^{2}$ o Nozick ${ }^{3}$, que son muy críticas de los derechos sociales y que defienden una versión minimalista de democracia. De otro lado, tenemos las concepciones cercanas al constitucionalismo social,

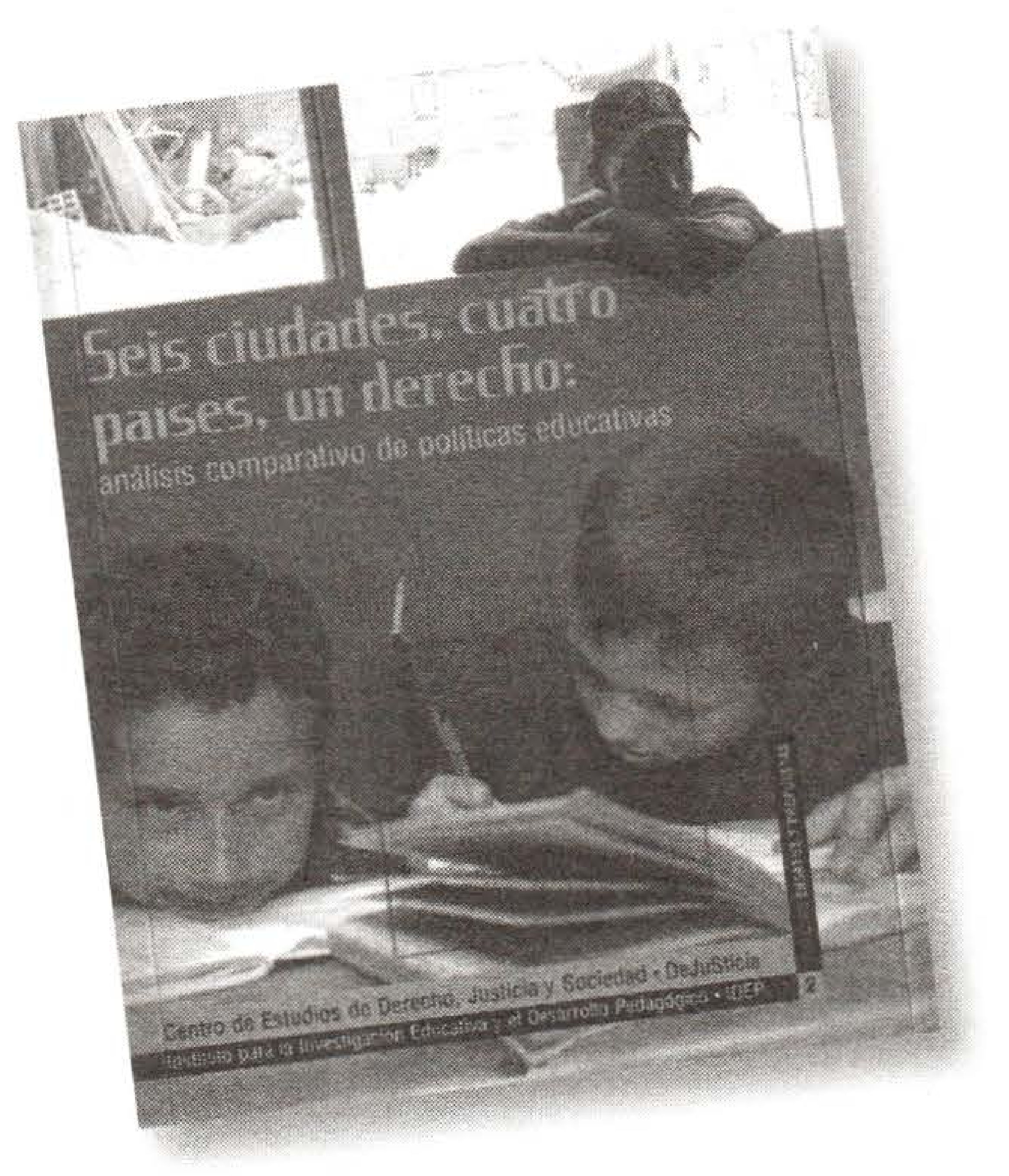

propias del llamado Estado social de rececho, que, por el contrario, buscan amparar no sólo los derechos individuales clásicos, como la intimidad o la libertad de expresión, sino también los derechos sociales como la educación o la salud, y que defienden entonces una visión más robusta y ampliada de democracia.

El Centro de Estudios de Derecho, Justicia y Sociedad DeJuSticia ${ }^{4}$ promueve, con base en estudios rigurosos, la formación de una ciudadanía sin exclusiones y la vigencia del Estado social de derecho, en especial en Colombia. Es pues natural que DeJuSticia adhiera a las concepciones que defienden la ciudadanía social y los derechos sociales y se oponga a las visiones minimalistas de la democracia y del Estado de derecho. Por ello, nuestro centro de estudios tiene como uno de sus ejes temáticos de trabajo la promoción y la defensa de los derechos sociales. Una de las formas como DeJuSticia

1 Apartes de la Presentación del libro: Pg. 9-15

2 Von Hayek, Friedrich, 1973, Law, legislation and liberty. Londres: Routledge and Kegan Paul.

3 Nozick, Robert. Anarquía, Estado y utopia. México: Fondo de Cultura Económica, 1988.

4 http://dejusticia.org/ 
promueve estos derechos es buscando que exista un mayor diálogo entre la formulación de las políticas públicas y el respeto y garantía de los derechos sociales, a fin de que esos derechos no sean vistos como "variables externas" al análisis de las políticas económicas y de las estrategias de desarrollo, sino que sean incorporados como una dimensión interna de las mismas.

Esta investigación buscó analizar la política educativa de Bogota con una perspectiva comparada y de derechos humanos, puesto que este enfoque armonizaba con el esfuerzo de DeJuSticia para poner en diálogo las políticas públicas con las exigencias normativas derivadas de los derechos sociales. El libro examina las políticas públicas de Bogotá, Medellín, Cartagena (Colombia; Buenos Aires (Brasil), Sao Paulo (Brasil) y Chile.

A manera de síntesis ${ }^{5}$ vale señalar que las experiencias de las ciudades y los resultados de sus políticas educativas contiene importantes enseñanzas el menos en dos sentidos. Primero, aunque las políticas que inciden en la eliminación de las barreras económicas para el disfrute del derecho a la educación son urgentes en nuestras ciudades y pueden incidir en la permanencia escolar, el derecho a tener una educación que se adapte a las necesidades de los individuos y de la sociedad no se agota en este ámbito. El papel de la educación en los niveles físico, emocional, intelectual y ético de las personas, y la complejidad del fenómeno de la deserción exigen políticas específicas en materia de permanencia que prevengan y corrijan el abandono escolar de manera integral.

Segundo, la ausencia de un enfoque de derechos humanos en las políticas públicas, bien reflejado en la carencia de estadísticas y políticas educativas para sujetos con necesidades educativas especiales, así como la falta de evaluación e impacto de las existentes, tienen consecuencias muy graves para la educación. Máxime cuando estas pertenecen a grupo vulnerables como desplazados, los niños y las niñas con talentos excepcionales o discapacidades físicas. Mientras se de preferencia a los enfoques de capital humano y desarrollo (en su acepción tradicional), las personas que tienen menores posibilidades de dinamizar y elevar las capacidades del mercado no serán una preocupación especial de las políticas públicas. Por eso, es primordial que los gobiernos incorporen un entendimiento de la educación como derecho de todas las personas y una obligación estatal que se robustece tratándose de los grupos más inermes de la sociedad.” 


\section{Los derechos sociales en serio: hacia un diálogo entre derechos y políticas públicas ${ }^{1}$}

Luis Eduardo Pérez Murcia, César Rodríguez Garavito y Rodrigo Uprimmy Yepes.

Bogotá, D.C.: Centro de Estudios de Derecho, Justicia y Sociedad DeJusticia, Instituto para la Investigación Educativa y el Desarrollo Pedagógico IDEP, 3. Ediciones Antropos, 2007.

"Este libro explora algunas de las relaciones existentes entre las políticas públicas y los derechos humanos. El estudio sostiene la tesis de que las políticas públicas constituyen un complemento ideal de los esfuerzos que hacen los jueces por prevenir, proteger y realizar los derechos humanos, y que, a su vez, los derechos humanos establecen límites normativos para la elaboración y aplicación de las políticas públicas en una democracia constitucional.

La investigación pone en evidencia que el fortalecimiento del vínculo entre derechos humanos y políticas públicas contribuye tanto al cumplimiento de las obligaciones del Estado como a la tarea de planeación con fundamento en el principio de la racionalidad económica. En otros términos, garantizar que las políticas públicas se diseñen, implementen y evalúen conforme con los contenidos de los derechos y las obligaciones del
Estado no sólo contribuye a mejorar el disfrute de los derechos humanos, sino que a la vez es la inversión más rentable para darles el mejor uso alternativo a los recursos escasos. Puntualmente, si el Estado asegura que las políticas públicas tengan un impacto positivo sobre los derechos, evitará ampliar las inversiones en el sistema de justicia que serían necesarias para responder al crecimiento de las demandas de la población por incumplimiento del os derechos y las responsabilidades del Estado.

Este texto defiende la importancia de que exista un mayor diálogo entre quines promueven los derechos humanos, y en especial los derechos sociales, y quienes elaboran las políticas públicas. Los artículos de este libro muestran, en fin, que es imperativo tender puentes entre la razón normativa de los derechos y la lógica técnica y económica en la discusión política de las estrategias de

Apartes de la Introducción del libro. Pg. 17-21.

2 http://dejusticia.org/archivo.php?id_tipo_publicacion=5, consultado el 9 de septiembre de 2008. 
desarrollo y en la formulación de las políticas públicas. Esos puentes son indispensables para la consolidación de las democracias vigorosas e incluyentes en Colombia y en América Latina".

"A partir de un análisis interdisciplinario sobre las relaciones conceptuales y prácticas entre los DESC y las políticas públicas, este libro sostiene que la protección de los derechos no incumbe sólo a los jueces y tiende puentes entre la razón normativa de los derechos y la lógica técnica y económica de las políticas públicas. Para ello, el libro indaga la aplicación de varios derechos sociales, con énfasis en el derecho a la educación" Esta publicación examina la relación entre el modelo económico y las políticas públicas, examinando el caso de la gratuidad de la educación primaria. Establece además, un modelo de indicadores para monitorear y evaluar el disfrute del derecho a la educación. 